Cite this article as: BMJ, doi:10.1136/bmj.38124.682523.55 (published 2 June 2004)

\title{
Papers
}

\section{Prospective cohort study of retinal vessel diameters and risk of hypertension}

Tien Yin Wong, Anoop Shankar, Ronald Klein, Barbara E K Klein, Larry D Hubbard

\begin{abstract}
Objective To examine the relation between diameters of the retinal arterioles and 10 year incidence of hypertension.

Design Population based prospective cohort study.

Setting Beaver Dam eye study.

Participants 2451 normotensive people aged 43 to 84 years. Main outcome measures Diameters of retinal arterioles and venules measured from digitised photographs of the retina taken at baseline. Measurements summarised as the arteriole:venule ratio, with a lower ratio indicating smaller arteriolar diameters. Incident hypertension, defined as systolic blood pressure $\geq 140 \mathrm{~mm} \mathrm{Hg}$, diastolic blood pressure $\geq 90$ $\mathrm{mm} \mathrm{Hg}$, or use of antihypertensive drugs during follow up. Results 721 participants developed hypertension over a 10 year period. Those with lower arteriole:venule ratio had a higher cumulative incidence of hypertension (incidences of $17.4 \%$, $24.1 \%, 31.0 \%$, and $45.1 \%$, respectively, for decreasing quarters of distribution of arteriole:venule ratio). After adjustment for age and sex, participants with arteriole:venule ratios in the lowest quarter had a threefold higher risk of hypertension (odds ratio 2.95, 95\% confidence interval 2.77 to 3.88) than those with ratios in the highest quarter. This association remained significant after further adjustment for baseline systolic and diastolic blood pressure and other risk factors (1.82, 1.39 to 2.40, for lowest versus highest ratio quarters).

Conclusions Narrowed retinal arterioles are associated with long term risk of hypertension, suggesting that structural alterations of the microvasculature may be linked to the development of hypertension.
\end{abstract}

\section{Introduction}

Contemporary guidelines for the treatment of hypertension are based on an empirical approach to lowering blood pressure, with less emphasis on specific antihypertensive treatment. ${ }^{1}$ In part, this is because of uncertainty about the basic pathophysiological mechanisms of essential hypertension. ${ }^{2}$

A key pathological characteristic of hypertension is the presence of small vessel disease, specifically vasoconstriction and narrowing of the peripheral small arteries and arterioles. ${ }^{3-7}$ Narrowing of these arterioles may contribute to the pathogenesis of hypertension by increasing peripheral vascular resistance. However, it is unclear whether arteriolar narrowing precedes development of hypertension or is merely a physiological response of raised blood pressure. ${ }^{8}$ There have been no long term prospective data showing a link between arteriolar narrowing and the subsequent risk of hypertension. ${ }^{8}$
Measurement of the retinal arterioles offers a non-invasive means to evaluate systemic associations of the human microcirculation in vivo. We have recently developed a computer based method to measure retinal arterioles and venules from photographic images. ${ }^{9}$ We examined the prospective association between retinal arteriolar diameters and the 10 year incidence of hypertension in a population based cohort of normotensive people.

\section{Methods}

The Beaver Dam eye study has been described in detail elsewhere ${ }^{10-12}$ In brief, a private census of the population of Beaver Dam, Wisconsin, USA, was performed in 1987-8. Ninety nine percent of the population is white. Of 5924 eligible individuals, 4926 participated in the baseline examination in $1988-90,{ }^{10} 3684$ $(81.1 \%$ of survivors) participated in the five year follow up examination in $1993-5,{ }^{11}$ and $2764(82.9 \%)$ participated in the 10 year follow up examination in 1998-2000. ${ }^{12}$ Comparisons between participants and non-participants at each of these examinations have been reported elsewhere. ${ }^{10-12}$ Informed consent was obtained from all participants.

To study the association between retinal vessel diameters and incident hypertension we excluded those with pre-existing hypertension at baseline $(\mathrm{n}=1820)$, missing systolic or diastolic blood pressure values $(\mathrm{n}=13)$, missing important covariate information (cigarette smoking $(\mathrm{n}=11)$, glycosylated haemoglobin concentrations $(n=14)$, physical activity $(n=16)$, total or high density lipoprotein cholesterol concentrations $(\mathrm{n}=34)$ ), or ungradeable retinal photographs $(n=295)$, leaving 2451 normotensive individuals who contributed data to the current analysis.

\section{Measurement of retinal vessels}

Methods used to measure and summarise retinal vessel diameters from digitised photographs followed a standardised protocol described elsewhere. ${ }^{13}{ }^{14}$ In brief, $30^{\circ}$ colour retinal photographs of the right eye taken at baseline were digitised by a high resolution scanner with standard settings. Graders masked to the characteristics of participants used a computer program to measure diameters of all arterioles and venules in a specified zone surrounding the optic disc. These measurements were combined into summary indices-the central retinal arteriolar and venular equivalents-which represented the average arteriolar and venular diameters of that eye, respectively. ${ }^{14}$ These were finally expressed as the retinal arteriole:venule ratio. The ratio compensated for possible magnification differences between eyes, and an arteriole:venule ratio of 1 indicated that arteriolar diameters were, on average, the same as venular diam- 


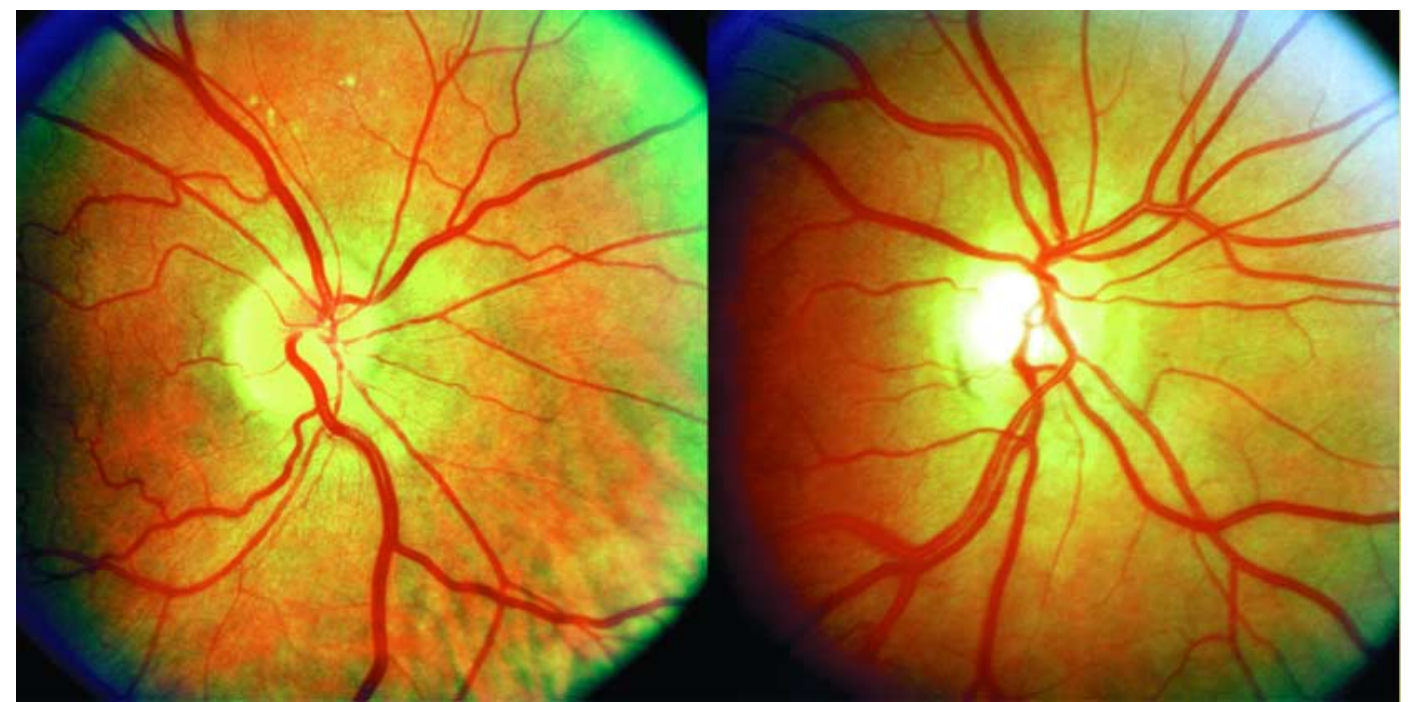

Fig 1 Retinal photographs showing normal retinal arterioles with arteriole:venule ratio of 1.08 (left) and narrowed retinal arterioles with arteriole:venule ratio of 0.64 (right)

eters in that eye, while a smaller ratio suggested narrower arterioles. ${ }^{14}$ Figure 1 shows examples of small (narrowed arterioles) and large (normal arterioles) ratios. Reproducibility of these retinal measurements has been previously reported, with intraclass correlation coefficients between and within graders ranging from 0.78 to $0.99 .{ }^{14}$

\section{Definitions of hypertension}

A standardised interview and examination was performed at each examination. ${ }^{15}$ Blood pressure was measured with a random zero sphygmomanometer according to the hypertension detection and follow up programme protocol at each examination. ${ }^{16}$ We used the average of two measurements for analysis.

We defined hypertension as systolic blood pressure $\geq 140$ $\mathrm{mm} \mathrm{Hg}$, diastolic blood pressure $\geq 90 \mathrm{~mm} \mathrm{Hg}$, or the combination of self reported diagnosis of high blood pressure and use of antihypertensive drugs. Participants who met these criteria at either the five year or 10 year follow up examination were defined as having incident hypertension.

\section{Definitions of other variables}

Non-fasting blood specimens were obtained from all participants. ${ }^{15}$ Plasma concentration of glycosylated haemoglobin was determined by using affinity chromatography, and serum concentrations of total cholesterol and high density lipoprotein cholesterol were determined by using enzymatic methods. We defined participants as having diabetes mellitus if they had a history of diabetes or diabetes had been newly diagnosed (no medical history of diabetes mellitus in the presence of raised glycosylated haemoglobin concentration and a random blood sugar $>11 \mathrm{nmol} / \mathrm{l}) .{ }^{15}$ Whenever the diagnosis was in doubt we consulted primary care physicians. We defined body mass index as weight $(\mathrm{kg}) /$ height $(\mathrm{m})^{2}$. Questions were asked relating to family income, education, physical activity, cigarette smoking, and alcohol consumption. For alcohol consumption, one serving was defined as 12 ounces $(0.46 \mathrm{l})$ of beer, 4 ounces $(0.12 \mathrm{l})$ of wine, or 1.5 ounces $(0.04 \mathrm{l})$ of liquor or distilled spirits. The amounts of alcohol from beer, wine, and liquor were summed to obtain the average ounces of alcohol consumed from any source in a week.

\section{Statistical analysis}

We categorised retinal vessel data (retinal arteriole:venule ratio, retinal arteriolar, and venular diameters) into quarters, with lower quarters indicating smaller ratios and vessel diameters. We also analysed data on retinal vessels as continuous variables (for example, per SD change in arteriole:venule ratio). We used logistic regression models to determine the odds ratio for incident hypertension, controlling simultaneously for possible confounders. We constructed three models, the first adjusting for age and sex; the second, additionally adjusting for diabetes, glycosylated haemoglobin concentration, body mass index, physical activity (number of times a week), total and high density lipoprotein cholesterol concentrations, and cigarette smoking (pack years smoked), and alcohol consumption (ounces per week); and the third, further adjusting for baseline systolic blood pressure, diastolic blood pressure, and pulse pressure. We repeated these analyses by using linear regression models of the retinal arteriole:venule ratio and the percentage change in systolic and diastolic blood pressure (log transformed) between baseline and the 10 year examination.

To examine the combined effect of baseline blood pressure and retinal arteriole:venule ratios on the risk of incident hypertension, we stratified the cohort into eight groups by baseline blood pressure ("normal" blood pressure and "prehypertension") and retinal arteriole:venule ratio quarters. "Normal" baseline blood pressure was defined as systolic $<120 \mathrm{~mm} \mathrm{Hg}$ and diastolic $<80 \mathrm{~mm} \mathrm{Hg}$ and "prehypertension" as systolic 120-139 $\mathrm{mm} \mathrm{Hg}$ or diastolic 80-90 $\mathrm{mm} \mathrm{Hg}$, according to the seventh joint national committee on the prevention, detection, evaluation, and treatment of high blood pressure classification. ${ }^{17}$

\section{Results}

Table 1 shows baseline characteristics of participants, according to arteriole:venule ratio. Lower arteriole:venule ratios were associated with older age, male sex, diabetes, higher glycosylated haemoglobin concentration, higher systolic and diastolic blood pressure, higher body mass index, higher total cholesterol concentration, lower high density lipoprotein cholesterol concentration, cigarette smoking, and alcohol consumption.

Over the 10 year period 721 people developed hypertension. After adjustment for age and sex, those who developed incident hypertension had significantly lower mean baseline arteriole:venule ratio $(0.69 v 0.72, \mathrm{P}<0.001)$ and smaller retinal arteriolar diameter $(166.0 \mu \mathrm{m} v 172.5 \mu \mathrm{m}, \mathrm{P}<0.001)$ compared with those 
Table 1 Baseline characteristics of study population in relation to retinal arteriole:venule ratio. Figures are means unless stated otherwise

\begin{tabular}{|c|c|c|c|c|c|}
\hline & \multicolumn{5}{|c|}{ Quarter of retinal arteriole:venule ratio* } \\
\hline & $1(n=612)$ & $2(\mathrm{n}=612)$ & $3(n=613)$ & $4(n=614)$ & P value \\
\hline Age (years) & 62.1 & 58.9 & 59.9 & 59.6 & $<0.001$ \\
\hline No $(\%)$ of men & $295(48.2)$ & $288(47.1)$ & $280(45.7)$ & $265(43.1)$ & $<0.001$ \\
\hline Family income (\$10 000‡) & 5.0 & 5.1 & 5.0 & 5.1 & 0.07 \\
\hline Length of schooling (years) & 12.0 & 12.2 & 12.9 & 12.2 & 0.08 \\
\hline No (\%) with diabetes & $43(7.1)$ & $38(6.2)$ & $33(5.4)$ & $31(5.1)$ & $<0.001$ \\
\hline Glycosylated haemoglobin (\%) & 6.7 & 5.9 & 5.7 & 5.4 & $<0.001$ \\
\hline Systolic blood pressure (mm Hg) & 129.2 & 127.1 & 122.8 & 120.2 & $<0.001$ \\
\hline Diastolic blood pressure $(\mathrm{mm} \mathrm{Hg})$ & 77.3 & 75.4 & 75.2 & 72.0 & $<0.001$ \\
\hline Body mass index $\left(\mathrm{kg} / \mathrm{m}^{2}\right)$ & 28.4 & 28.2 & 28.1 & 26.2 & 0.05 \\
\hline Total cholesterol (mmol/l) & 6.0 & 5.9 & 5.9 & 5.9 & 0.05 \\
\hline HDL cholesterol $(\mathrm{mmol} / \mathrm{l})$ & 1.3 & 1.4 & 1.4 & 1.4 & 0.06 \\
\hline Cigarette smoking (pack years) & 20.8 & 18.9 & 18.1 & 14.2 & $<0.001$ \\
\hline Alcohol intake (ounces/week)§ & 4.8 & 4.4 & 4.1 & 3.4 & 0.05 \\
\hline
\end{tabular}

*Ranges: $1 \mathrm{st}=0.50-0.67,2 \mathrm{nd}=0.68-0.71,3 \mathrm{rd}=0.72-0.75,4 \mathrm{th}=0.76-1.04$.

†For test of difference in characteristics by arteriole:venule ratio quarters based on analysis of variance or $\chi^{2}$ tests as appropriate. $\ddagger £ 5684(€ 8413)$.

$\S$ See text.

who did not develop hypertension. In contrast, baseline retinal venular diameter was similar in participants who did and did not develop hypertension $(241.0 \mu \mathrm{m} v 241.7 \mu \mathrm{m}, \mathrm{P}=0.17)$.

Table 2 shows the incidence and odds ratio for incident hypertension in relation to the retinal arteriole:venule ratio. After we controlled for age and sex, participants with an arteriole:venule ratio in the first (lowest) quarter were three times as likely to develop hypertension than those with a ratio in the fourth quarter (odds ratio $2.95,95 \%$ confidence interval 2.27 to 3.85 ). Further adjustment for risk factors associated with hypertension had minimal impact on this association (2.72, 2.08 to 3.54). This association was attenuated but remained significant even after further adjustment for baseline systolic and diastolic blood pressure and pulse pressure (1.82, 1.39 to 2.40$)$. The pattern of association for retinal arteriolar diameter was essentially similar to that of the arteriole:venule ratio, but retinal venular diameter was unrelated to incident hypertension (see table A on bmj.com).

When we stratified data according to baseline blood pressure, participants who were classified as "pre-hypertensive" and who had lower arteriole:venule ratios were more likely to develop hypertension than people with "normal" baseline blood pressure and higher arteriole:venule ratio (fig 2).

When we analysed the arteriole:venule ratio as a continuous variable, each SD decrease (a decrease of 0.07 ) was associated with a $30 \%$ increase in odds of hypertension (table 3). We examined for possible interaction with sex and other risk factors for hypertension (diabetes, body mass index, waist:hip ratio, cigarette smoking, and alcohol consumption) by stratification (table 3) and by inclusion of cross product terms in the logistic regression models. We did not find significant interactions (all cross product terms $\mathrm{P}>0.20$ ).

Finally, we constructed multiple linear regression models of retinal arteriole:venule ratio and change in blood pressure between baseline and the 10 year follow up. After we excluded participants taking antihypertensive medications at the follow up examinations, baseline retinal arteriole:venule ratio was inversely associated with a 10 year change in blood pressure (see table B on bmj.com). When we included the blood pressures of those who were taking antihypertensive medications at the follow up examinations, the magnitude of this inverse association was attenuated, as expected, but remained significant $(\mathrm{P}<0.05)$.

\section{Discusssion}

In this prospective cohort study we showed that smaller retinal arteriolar diameter is associated with increased risk of hypertension over a 10 year period. After we controlled for age, sex, preexisting systolic and diastolic blood pressure, diabetes, body mass index, and other risk factors for hypertension, people with an arteriole:venule ratio in the lowest quarter were about $80 \%$ more likely to develop hypertension than those with a ratio in the highest quarter. Furthermore, each SD reduction in the arteriole:venule ratio was associated with a $30 \%$ increased risk of hypertension, independent of baseline blood pressure and other risk factors.

Experimental studies have long indicated that peripheral arteriolar constriction and increased vascular resistance are classic features of essential hypertension. Narrowed small arterioles may cause hypertension by increasing peripheral vascular resist-

Table 2 Odds ratio (95\% confidence interval) for incident hypertension in relation to retinal arteriole:venule ratio

\begin{tabular}{|c|c|c|c|c|c|}
\hline \multirow[b]{2}{*}{ Ratio } & \multirow[b]{2}{*}{ No at risk } & \multicolumn{4}{|c|}{ Incident hypertension } \\
\hline & & No of cases & Adjusted for age and sex & Multivariable* & Multivariable $\dagger$ \\
\hline 1st quarter & 612 & 276 & 2.95 (2.27 to 3.85$)$ & 2.72 (2.08 to 3.54 ) & 1.82 (1.39 to 2.40$)$ \\
\hline 2nd quarter & 612 & 190 & 2.05 (1.57 to 2.69 ) & 1.91 (1.46 to 2.51 ) & 1.47 (1.11 to 1.95$)$ \\
\hline 3rd quarter & 613 & 148 & 1.17 (0.87 to 1.55$)$ & $1.13(0.85$ to 1.51$)$ & 0.98 (0.73 to 1.32$)$ \\
\hline 4th quarter & 614 & 107 & $1 \ddagger$ & $1 \neq$ & $1 \ddagger$ \\
\hline $1 \mathrm{SD}$ decrease & 2451 & 721 & 1.54 (1.38 to 1.72$)$ & 1.50 (1.34 to 1.68$)$ & 1.31 (1.18 to 1.45$)$ \\
\hline
\end{tabular}

${ }^{*}$ Further adjusted for diabetes, glycosylated haemoglobin, body mass index, physical activity, total and high density lipoprotein cholesterol concentrations, cigarette smoking, and alcohol consumption.

†Further adjusted for systolic and diastolic blood pressure and pulse pressure.

$\ddagger$ Reference. 


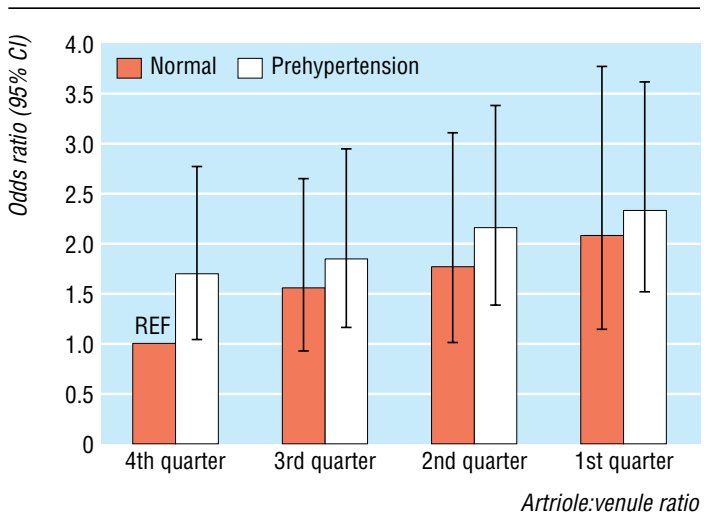

Fig 2 Odds ratios for incident hypertension associated with baseline blood pressure (normal, prehypertensive) and arteriole:venule ratio quarters, adjusted for variables listed in table 3 . Reference group (REF) is normal blood pressure and fourth (highest) quarter of arteriole:venule ratio

ance and haemodynamic load. However, partly because of a lack of prospective studies, there is no clear consensus as to whether reduced arteriolar size arises as an adaptive consequence of raised blood pressure or is a pathogenic factor in the development of hypertension. ${ }^{1-9}$ Our study now provides longitudinal data in a large unselected cohort of normotensive people to support the concept that arteriolar narrowing precedes the subsequent development of hypertension.

Narrowing of the retinal arterioles seen clinically reflects intimal thickening and medial hyperplasia, hyalinisation, and sclerosis seen histopathologically. ${ }^{18}$ We have previously shown that such narrowing is not only associated with raised blood pressure but also with systemic markers of inflammation and possibly endothelial dysfunction. ${ }^{19}$ It is therefore possible that microvascular narrowing due to these mechanisms is involved in the pathogenesis of hypertension.

Table 3 Odds ratio (95\% confidence interval) for incident hypertension per SD decrease in retinal arteriole:venule ratio, stratified by risk factors

\begin{tabular}{|c|c|c|c|}
\hline & \multirow[b]{2}{*}{ No at risk } & \multicolumn{2}{|c|}{ Incident hypertension } \\
\hline & & No of cases & $\begin{array}{c}\mathrm{OR}^{*}(95 \% \mathrm{CI}) \text { per SD } \\
\text { decrease in ratio }\end{array}$ \\
\hline All & 2451 & 721 & 1.31 (1.18 to 1.45$)$ \\
\hline \multicolumn{4}{|l|}{ Sex: } \\
\hline Men & 1077 & 294 & 1.52 (1.28 to 1.87$)$ \\
\hline Women & 1367 & 427 & 1.19 (1.04 to 1.36$)$ \\
\hline \multicolumn{4}{|l|}{ Diabetes: } \\
\hline No & 2303 & 663 & 1.31 (1.18 to 1.47$)$ \\
\hline Yes & 148 & 58 & 1.24 (0.85 to 1.92$)$ \\
\hline \multicolumn{4}{|c|}{ Body mass index $\left(\mathrm{kg} / \mathrm{m}^{2}\right)$ : } \\
\hline Low $(<28)$ & 1326 & 330 & 1.33 (1.13 to 1.58$)$ \\
\hline High $(\geq 28)$ & 1118 & 391 & 1.28 (1.10 to 1.49$)$ \\
\hline \multicolumn{4}{|l|}{ Waist:hip ratio: } \\
\hline Low $(<0.94)$ & 1738 & 491 & 1.28 (1.12 to 1.45$)$ \\
\hline $\operatorname{High}(\geq 0.94)$ & 706 & 230 & 1.38 (1.12 to 1.70$)$ \\
\hline \multicolumn{4}{|c|}{ Cigarette smoking: } \\
\hline Never & 1065 & 326 & 1.23 (1.06 to 1.43$)$ \\
\hline Previous & 832 & 251 & 1.33 (1.10 to 1.62) \\
\hline Current & 547 & 144 & 1.50 (1.16 to 2.01$)$ \\
\hline \multicolumn{4}{|c|}{ Current alcohol intake: } \\
\hline No & 280 & 86 & 1.07 (0.80 to 1.45$)$ \\
\hline Yes & 2164 & 635 & 1.34 (1.19 to 1.50$)$ \\
\hline
\end{tabular}

*Adjusted for age, sex, diabetes, glycosylated haemoglobin, body mass index, physical activity, total and high density lipoprotein cholesterol concentrations, cigarette smoking activity, total and high density lipoprotein cholesterol concentrations, cigarette smoking,
alcohol consumption, systolic and diastolic blood pressure, and pulse pressure in logistic alcohol consumption,
regression models.
There are few studies for direct comparison. The atherosclerosis risk in communities study also found that narrowed retinal arteriolar diameter was associated with an increased risk of hypertension developing over a three year period in 5628 normotensive people (multivariable adjusted relative risk 1.62, $95 \%$ confidence interval 1.21 to 2.18 , comparing lowest to highest fifths of arteriole:venule ratio). ${ }^{20}$ That study, however, was limited by a relatively short interval to follow up. We had a considerably longer follow up, and our results provide stronger evidence that reduced retinal arteriolar diameter precedes the long term risk of hypertension.

We have shown that combined exposure to higher pre-existing blood pressure at baseline and narrowed arterioles was associated with a higher risk of hypertension than the effect of either alone (fig 2). This supports the theory that higher blood pressure might cause arteriolar vasoconstriction, vascular remodelling, and greater peripheral vascular resistance, leading to further increases in blood pressure and the maintenance of the hypertensive state. ${ }^{21}$

\section{Limitations}

Several limitations of this study should be highlighted. Firstly, blood pressure measurements taken once during each five year interval may have resulted in some misclassification of hypertension. However, we have no reason to believe that potential misclassification error would substantially bias our results (except towards the null). Secondly, residual confounding from pre-existing hypertension or other unmeasured factors may explain some of these associations. For example, the strength of the association between arteriolar narrowing and risk of hypertension was substantially weakened after we adjusted for baseline blood pressure, suggesting that baseline blood pressure was an important confounder. Finally, selective mortality may have masked some associations. If people with reduced arteriolar diameters who were at risk of developing hypertension were more likely to die before the follow up examinations, these associations could be falsely attenuated. Strengths of our study include its population based nature, long follow up, quantitative evaluation of retinal arteriolar diameters, and standardised measurement of blood pressure.

\section{Clinical implications}

Our results may have potential clinical implications. There is increasing recognition of the value of specifically targeting the microcirculation in the prevention and treatment of hypertension and its complications. ${ }^{22}{ }^{23}$ For example, there is some evidence that angiotensin converting enzyme inhibitors have direct beneficial effects on microvascular structure and may have therapeutic value in preventing cardiovascular morbidity beyond that of lowering blood pressure. ${ }^{24}$ Our study indicates that further research to evaluate approaches to treatment focused on the microcirculation may be important.

In conclusion, our study shows a prospective association between reduced retinal arteriolar diameters and the 10 year incidence of hypertension in the general population. Arteriolar narrowing and factors related to this microvascular change may be linked to the development of hypertension.

These data were presented at the 44th Annual Conference on Cardiovascular Disease Epidemiology and Prevention, American Heart Association, San Francisco, March 2004.

Contributors: TYW formulated the hypothesis and wrote the initial and final drafts. AS performed the statistical analysis and provided additional intellectual input. RK and BEKK reviewed the manuscript and provided overall supervision and administrative support for the project. LDH designed the method of measuring retinal vessel diameters and critically reviewed the paper. TYW is guarantor. 


\section{What is already known on this topic}

Narrowing of the small blood vessels may have a role in the pathogenesis of hypertension, though few prospective trials support such a hypothesis

\section{What this study adds}

People with smaller retinal arteriolar diameters are more likely to develop hypertension than people with larger arteriolar diameters, independent of known risk factors for hypertension

This finding suggests that narrowed arterioles are associated with the development of hypertension and therefore that small vessel disease may be a target for antihypertensive treatment

Funding: American Diabetes Association (RK, AS), NIH grants EYO6594 (RK, BEKK) and HL66018 (RK, TYW), and the Singapore Biomedical Research Council Young Investigator Award (TYW).

Competing interests: None declared.

Ethical approval: All data were collected with Institutional Review Board approval in conformity with all country, federal, and state laws.

1 Mulrow CD, Pignone M. What are the elements of good treatment for hypertension? BMJ 2001;322:1107-9.

2 Beevers G, Lip GY, O'Brien E. ABC of hypertension: the pathophysiology of hypertension. BMJ 2001;322:912-6.

3 Folkow B. Physiological aspects of primary hypertension. Physiol Rev 1982;62:347-504

4 Mulvany MJ, Aalkjaer C. Structure and function of small arteries. Physiol Rev Mulvany MJ, Aalkjaer C. Structure and function of small arteries. Physiol Res 1990;70:921-61.

5 Rizzoni D, Castellano M, Porteri E, Bettoni G, Muiesan ML, Agabiti-Rosei E. Vascular structural and functional alterations before and after the development of hypertension in SHR. Am J Hypertens 1994;7:193-200.

6 Norrelund H, Christensen KL, Samani NJ, Kimber P, Mulvany MJ, Korsgaard N. Early narrowed afferent arteriole is a contributor to the development of hypertension. Hypertension 1994;24:301-8.

7 Mulvany MJ, Baumbach GL, Aalkjaer C, Heagerty AM, Korsgaard N, Schiffrin EL, et al. Vascular remodeling. Hypertension 1996;28:505-6.

8 Mulvany MJ. Are vascular abnormalities a primary cause or secondary consequence of hypertension? Hypertension 1991;18(3 suppl):I52-7.

9 Hubbard LD, Brothers RJ, King WN, Clegg LX, Klein R, Cooper LS, et al. Methods for evaluation of retinal microvascular abnormalities associated with hypertension/ sclerosis in the atherosclerosis risk in communities (ARIC) study. Ophthalmology 1999;106:2269-80.
10 Klein R, Klein BEK, Linton KLP, DeMets DL. The Beaver Dam eye study: visual acuity Ophthalmology 1991;98:1310-5.

11 Klein R, Klein BEK, Lee KE. The changes in visual acuity in a population. The Beaver Dam eye study. Ophthalmology 1996;103:1169-78.

12 Klein R, Klein BE, Lee KE, Cruickshanks KJ, Chappell RJ. Changes in visual acuity in a population over a 10-year period: the Beaver Dam eye study. Ophthalmology. 2001;108:1757-66

13 Wong TY, Klein R, Klein BEK, Meuer SM, Hubbard LD. Retinal vessel diameters and their associations with age and blood pressure. Invest Ophthalmol Vis Sci 2003;44:4644-

14 Knudtson MD, Lee KE, Hubbard LD, Wong TY, Klein R, Klein BEK. A revised method for summarizing retinal vessel diameters. Curr Eye Res 2003;27:143-9.

15 Klein R, Klein BEK. Beaver Dam eye study. Manual of operations. Springfield, VA: Department of Ophthalmology, University of Wisconsin-Madison, 1991. (NTIS Accession No. PB 91-149823.)

16 Hypertension Detection and Follow-up Program Cooperative Group. The hypertension detection and follow-up program. Prev Med 1976;5:207-15.

17 Chobanian AV, Bakris GL, Black HR, Cushman WC, Green LA, Izzo JL Jr, et al. The seventh report of the joint national committee on prevention, detection, evaluation, and treatment of high blood pressure: the JNC 7 report. JAMA 2003;289:2560-71.

18 Tso MOM, Jampol LM. Pathophysiology of hypertensive retinopathy. Ophthalmology 1982;89:1132-45.

19 Klein R, Sharrett AR, Klein BE, Chambless LE, Cooper LS, Hubbard LD, et al. Are retinal arteriolar abnormalities related to atherosclerosis? The atherosclerosis risk in communities study. Arterioscler Thromb Vasc Biol 2000;20:1644-50.

20 Wong TY, Klein R, Sharrett AR, Duncan BB, Couper DJ, Klein BEK. Retinal arteriolar diameters and risk of hypertension. Ann Intern Med 2004;140:248-55.

21 Pries AR, Secomb TW, Gaehtgens P. Structural autoregulation of terminal vascular beds: vascular adaptation and development of hypertension. Hypertension 1999:33:15361.

22 Thybo NK, Stephens N, Cooper A, Aalkjaer C, Heagerty AM, Mulvany MJ, et al. Effect of antihypertensive treatment on small arteries of patients with previously untreated essential hypertension. Hypertension 1995;25:474-81.

23 Rizzoni D, Porteri E, Boari GE, De Ciuceis C, Sleiman I, Muiesan ML, et al. Prognostic significance of small-artery structure in hypertension. Circulation 2003;108:2230-5.

24 Levy BI, Ambrosio G, Pries AR, Struijker-Boudier HA. Microcirculation in hypertension: a new target for treatment? Circulation 2001;104:735-40.

(Accepted 29 April 2004)

doi $10.1136 / \mathrm{bmj} .38124 .682523 .55$

Centre for Eye Research Australia, University of Melbourne, Victoria 3002, Australia

Tien Yin Wong associate professor

Department of Ophthalmology, University of Wisconsin, Madison, Wisconsin 53726, USA

Anoop Shankar research associate

Ronald Klein professor

Barbara E K Klein professor

Fundus Photograph Reading Centre, University of Wisconsin, Madison

Larry D Hubbard associate director

Correspondence to: T Wong twong@unimelb.edu.au 